\title{
Hepatitis E virus chronic infection of swine co-infected with Porcine Reproductive and Respiratory Syndrome Virus
}

Morgane Salines ${ }^{1,2+}$, Elodie Barnaud ${ }^{3,4,5+}$, Mathieu Andraud ${ }^{1,2}$, Florent Eono ${ }^{1,2}$, Patricia Renson ${ }^{1,2}$, Olivier Bourry ${ }^{1,2}$, Nicole Pavio ${ }^{3,4,5}$ and Nicolas Rose $e^{1,2^{*}}$

\begin{abstract}
In developed countries, most of hepatitis E human cases are of zoonotic origin. Swine is a major hepatitis E virus (HEV) reservoir and foodborne transmissions after pork product consumption have been described. The risk for HEV-containing pig livers at slaughter time is related to the age at infection and to the virus shedding duration. Porcine Reproductive and Respiratory Syndrome Virus (PRRSV) is a virus that impairs the immune response; it is highly prevalent in pig production areas and suspected to influence HEV infection dynamics. The impact of PRRSV on the features of HEV infections was studied through an experimental HEV/PRRSV co-infection of specific-pathogen-free (SPF) pigs. The follow-up of the co-infected animals showed that HEV shedding was delayed by a factor of 1.9 in co-infected pigs compared to HEV-only infected pigs and specific immune response was delayed by a factor of 1.6. HEV shedding was significantly increased with co-infection and dramatically extended (48.6 versus 9.7 days for HEV only). The long-term HEV shedding was significantly correlated with the delayed humoral response in co-infected pigs. Direct transmission rate was estimated to be 4.7 times higher in case of co-infection than in HEV only infected pigs ( 0.70 and 0.15 per day respectively). HEV infection susceptibility was increased by a factor of 3.3 , showing the major impact of PRRSV infection on HEV dynamics. Finally, HEV/PRRSV co-infection - frequently observed in pig herds - may lead to chronic HEV infection which may dramatically increase the risk of pig livers containing HEV at slaughter time.
\end{abstract}

\section{Introduction}

Hepatitis E virus is a non-enveloped single-stranded RNA virus causing an acute hepatitis in humans. It is mainly transmitted by the oro-fecal route and is responsible for clinical signs similar to hepatitis A virus infection [1]. Chronic cases have been described, mainly in immunocompromised patients [2,3]. Four HEV genotypes have been described. Genotypes 1 and 2 infect only humans and circulate in Asia, Africa and Central America in epidemic waves linked to the consumption of contaminated water [4-6]. Genotypes 3 and 4 are shared between humans and other animal species and are responsible for autochthonous sporadic cases in industrialized countries. In particular, the number of hepatitis $\mathrm{E}$ cases linked to genotype 3 has considerably increased in the last decade

\footnotetext{
* Correspondence: nicolas.rose@anses.fr

${ }^{\dagger}$ Equal contributors

'ANSES, Laboratoire de Ploufragan-Plouzané, BP 53, 22440 Ploufragan, France

¿Université européenne de Bretagne, 35000 Rennes, France

Full list of author information is available at the end of the article
}

[6,7], in relation to better diagnosis. This genotype is highly prevalent in the swine population [8]. Some studies have shown that swine and human HEV strains are genetically very close [9] and HEV cross-species transmission has been proven $[10,11]$. Moreover, a number of autochthonous cases have been related to the consumption of undercooked pork meat, especially liver products [12-16]. Thus, hepatitis $E$ is now recognized as a foodborne zoonosis for which domestic pigs are considered as the main reservoir in developed countries $[7,17,18]$. Understanding factors influencing the transmission dynamics of HEV in pig herds is crucial to limit the risk of an introduction of contaminated products in the food chain. Several studies have described experimental HEV infection trials via oral or intravenous route [19-24] but few studies were aimed at quantifying HEV transmission [20,25]. The results of these studies on HEV transmission were different than those observed in pig farms on the field, with the latent and infectious period estimates being generally longer

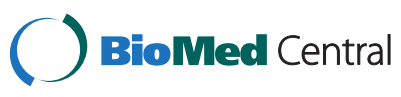

(C) 2015 Salines et al.; licensee BioMed Central. This is an Open Access article distributed under the terms of the Creative Commons Attribution License (http://creativecommons.org/licenses/by/4.0), which permits unrestricted use, distribution, and reproduction in any medium, provided the original work is properly credited. The Creative Commons Public Domain Dedication waiver (http://creativecommons.org/publicdomain/zero/1.0/) applies to the data made available in this article unless otherwise stated. 
than in experimental trials [26-28]. Moreover, a high variability of HEV infection dynamics is observed on pig farms and has not yet been fully explained [29]. Some factors affecting swine immune response may also influence the course of HEV infection. Porcine Respiratory and Reproductive Syndrome Virus (PRRSV) is a highly prevalent virus that impairs the immune response. It has been detected together with HEV in several studies but no evidence of a causal relationship has been shown to date [30-32]. Since chronic cases in humans are generally linked to immunosuppressive conditions [33-36], PRRSV might be suspected as a frequent co-factor affecting the features of HEV infection in pigs.

The impact of a PRRSV infection on HEV infection dynamics (in terms of viral shedding duration and quantity, transmission and humoral immune response) has therefore been studied through a transmission experiment involving HEV/PRRSV co-infection of specific-pathogenfree (SPF) pigs compared to an infection trial with HEV only that was previously led in our facilities, under the same conditions [25].

\section{Materials and methods}

HEV-only infection experiment

A transmission trial with HEV only has been carried out before the co-infection experiment [25]. The experiment was conducted in Anses air-filtered level-3 biosecurity facilities. Briefly, sixty-eight SPF Large-White piglets were used for the experiment. Eight pigs were kept as negative controls and the others were allocated to six rooms containing two pens per room. Rooms 1 to 3 were used to evaluate direct and environmental transmission, whereas Rooms 4 to 6 were used to examine between-pen transmission. The inoculated pigs received orally $10^{8}$ ge (genome equivalent) under a volume of $10 \mathrm{~mL}$ of a genotype $3 \mathrm{HEV}$ suspension (strain FR-SHEV3e, Genbank access number JQ953665). Individual fecal samples were collected four days before inoculation and three times per week from 0 to 39 days postinfection (dpi) when the pigs were killed for necropsy. Blood samples were collected twice a week during the same period and clinical signs and rectal temperature were monitored on a daily basis.

\section{HEV/PPRSV co-infection experiment Animal housing conditions and inoculation}

The experiment was conducted in the same Anses airfiltered level-3 biosecurity facilities. Twenty five-week-old SPF Large-White piglets were included in the study; they were HEV and PRRSV free and they did not have any maternal antibodies against these two viruses. Pigs were housed in metallic flat decks with a punched floor for feces and urine evacuation. As in the field situation, fecal material could accumulate in the corners and was not removed during the trial. Three rooms were used: two negative control pigs were housed in Room 1 whereas the 18 remaining piglets were randomly allocated to 3 independent pens distributed in Room 2 and Room 3 (6 piglets per pen) stratifying on gender ( 3 males and 3 females per pen), weight and the litter they came from. Room 2 contained 2 pens separated by a solid partition to prevent contamination of a pen by the other one (Figure 1). The average weights at weaning (sd) were $9.5 \mathrm{~kg}(2.7), 9.3 \mathrm{~kg}$ (1.6), $9.3 \mathrm{~kg}(2.3)$ and $9.3 \mathrm{~kg}$ (1.4) for Controls and groups \#1, \#2 and \#3 respectively. In each pen, 3 piglets were inoculated with both HEV and PRRSV at day 0. For inoculation, piglets to be inoculated were grouped in a pen and they were put in contact with their corresponding pen-mates at day 1 . The 3 inoculated piglets received the following: (i) orally $10^{8}$ ge under a volume of $10 \mathrm{~mL}$ of a genotype $3 \mathrm{HEV}$ suspension (strain FR-SHEV3e, Genbank access number JQ953665) prepared according to the protocol previously described in Andraud et al. [25] (ii) and by nasal route $2.5 \mathrm{~mL}$ per nostril of a PRRSV suspension (strain PRRS-2005-29-24-1 "Finistere", genotype 1, subtype 1) titrating $10^{5} \mathrm{TCID}_{50} / \mathrm{mL}$. The experiment was performed in accordance with EU and French regulations on animal welfare in experiments. The protocol was approved by the Anses/ENVA/UPEC ethical committee (agreement \#16 with the National committee for Ethics in animal experimentation).

\section{Data collection}

Individual fecal samples were collected three days before inoculation and three times a week until the end of the experiment ( $49 \mathrm{dpi})$. Blood samples were collected before inoculation and once a week until the end of the experiment. Clinical examination was also performed (clinical signs, rectal temperature, feces consistence, weight, food consumption and trough cleanliness were recorded daily). Euthanasia was carried out by intravenous injection of $1 \mathrm{~g} /$ $50 \mathrm{~kg}$ live weight of $\mathrm{Nesdonal}^{\circ}$ (thiopental-sodium, Merial, Lyon, France) followed by exsanguination. Necropsy was performed and liver samples were taken.

Because HEV is a zoonotic agent, strict biosecurity measures were applied to prevent any transmission from pigs to animal technicians.

\section{Virology and serology analyses}

HEV RNA quantification in fecal and liver samples was performed, after manual total RNA extraction, using realtime quantitative RT-PCR as described in Barnaud et al. [37] and Andraud et al. [25]. The results were expressed in terms of Cycle threshold (Ct). Standard quantification curves were produced by plotting the $\mathrm{Ct}$ values against the logarithm of the input copy numbers of standard RNA. Standard RNA was obtained after in vitro transcription of a plasmid pCDNA 3.1 ORF 2-3 HEV, as described in Barnaud et al. [37]. The results are expressed in genomic copy number per gram of feces (ge/g). 


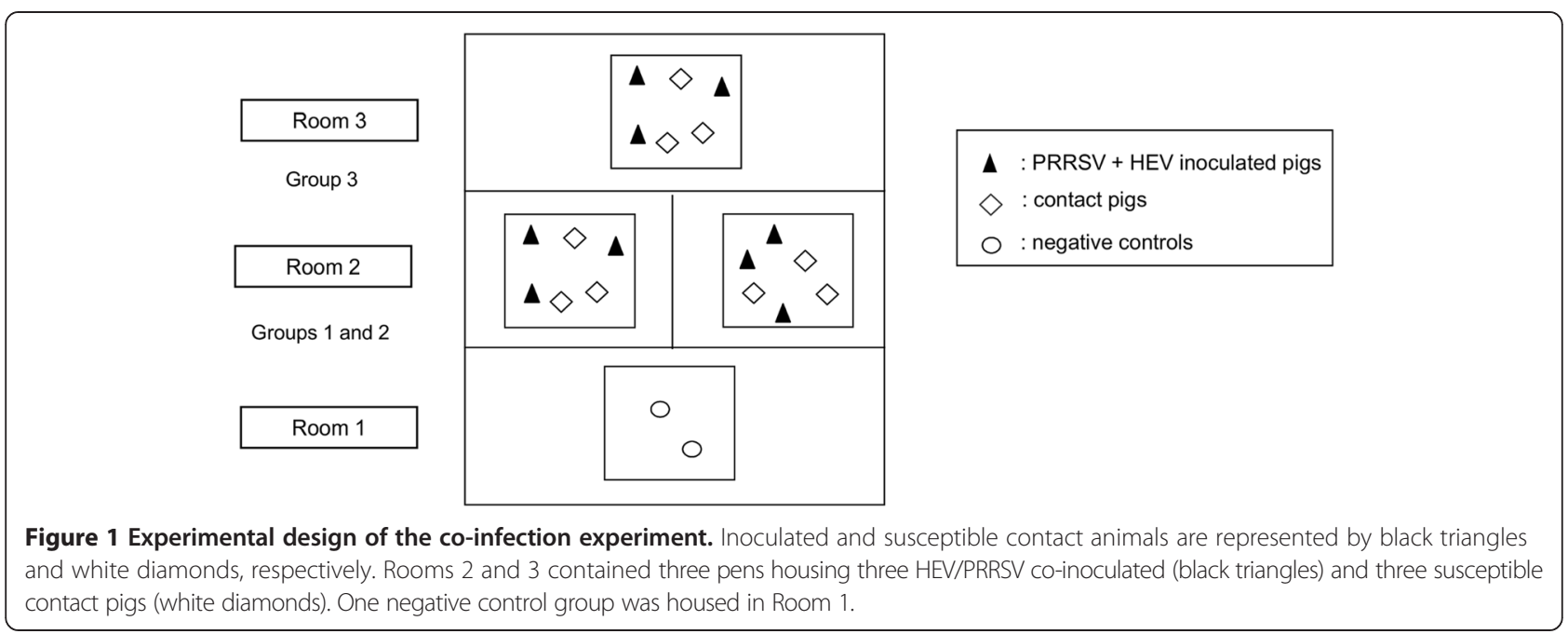

The detection of anti-HEV antibodies was performed using the HEV ELISA 4.0v kit (MP Diagnostics, Illkirch, France) according to the manufacturer's instructions, except the serum quantity used $(10 \mu \mathrm{L}$ instead of $20 \mu \mathrm{L}$ ). This sandwich ELISA allows the detection of all antibody classes (IgG, IgM and IgA) and uses a recombinant antigen that is present in all HEV strains. Samples were positive when the optical density at $450 \mathrm{~nm}$ wavelength obtained for the sample was higher than the threshold defined as the mean for negative controls +0.3 .

PRRSV RNA detection in sera was performed using a real-time RT-PCR as described in Charpin et al. [38]. Briefly, RNA extraction was performed using the NucleoSpin $^{\circ} 8$ virus kit (Macherey-Nagel, Düren, Germany) according to the manufacturer's instructions. RNA detection was then performed using the mix GoTaq 1-Step RT-qPCR System (Promega) supplemented with probes and specific primers of the target gene (ORF7 pan-PRRSV) and of the internal reference gene (swine Beta-Actin). The RT-PCR was performed on a Bio-Rad Chromo4 real-time PCR detection system (Bio-Rad) according to the following program: $50{ }^{\circ} \mathrm{C}$ for $30 \mathrm{~min}, 94{ }^{\circ} \mathrm{C}$ for 2 min followed by 45 cycles of $94{ }^{\circ} \mathrm{C}$ for $15 \mathrm{~s}$ and $60{ }^{\circ} \mathrm{C}$ for $30 \mathrm{~s}$. The results are expressed in $\mathrm{Ct}$.

\section{Models}

\section{Estimation of durations related to HEV infection dynamics}

The latent and infectious period durations and the duration of the period required to produce anti-HEV antibodies were estimated using survival data analyses. For each inoculated animal, the latent period was determined as the time elapsed between the inoculation day and the date of the first positive fecal sample for HEV RNA. The latent period after inoculation was fitted to a gamma distribution, from which the shape and scale parameters were estimated by the maximum log-likelihood method. A nonparametric bootstrap procedure was used to determine the $95 \%$ confidence interval of the parameter estimates.

A parametric model for survival data was built to estimate the duration of the infectious period, using the RT-PCR performed on livers after euthanasia as the last observation date. Two parametric models were tested (log-normal and Weibull distributions of survival times) and compared using the Akaike Information Criterion (AIC).

The impact of PRRSV co-infection on the time to HEV seroconversion was also studied with a parametric survival model applied to the data from the co-infection trial and the only HEV infection experiment [25]. The link between the earliness of the HEV antibody response and the duration of the infectious period was studied with a Cox model. The immune response was considered as absent or late if the delay before seroconversion was longer than $25 \mathrm{dpi}$, and as early if it was shorter than 25 dpi [39].

All analyses were performed using the R software (survreg and coxph functions) [40].

\section{Quantification of HEV shedding, environmental accumulation and transmission}

The distributions of HEV shed viral loads with time (with and without co-infection) are represented with box plot series. A linear mixed model (proc Mixed, SAS 9.3, [41]) which took into account repeated measurements with time was built to study the difference in the quantity of HEV shed particles between co-infected and non co-infected pigs.

The environmental load corresponds to the accumulation of viral particles in the environment through fecal shedding by infected animals, which is partially compensated by the clearance rate hereafter denoted $\delta$. The clearance rate takes into account feces elimination 
through the metallic flat deck and HEV intrinsic mortality in the environment. As described in Andraud et al. [25], for each pen $(k)$ and every sampling time $\left(t_{i}\right)$, the average quantity of genome equivalent shed in the environment per gram of feces was calculated with:

$$
V_{k}\left(t_{i}\right)=\sum_{j} V_{k}^{j}\left(t_{i}\right) / N_{k}
$$

where $V_{k}^{\dot{j}}\left(t_{i}\right)$ represents the quantity of virus shed per gram of feces in pen $k$ by pig $j$ at time $t_{i}$ and $N_{k}$ the total number of animals in pen $k$. Thus the cumulated viral load in the environment of pen $k$ between two sampling times $t_{i}$ and $t_{i+1}$ is given by the equation:

$$
\begin{aligned}
E_{k i} & =E_{k}\left(t_{i+1}\right) \\
& =\left(E_{k}\left(t_{i}\right)+\int_{0}^{\Delta t} V_{k}\left(t_{i}+u\right) e^{\delta u} d u\right) e^{-\delta \Delta t}, \text { with } \Delta t \\
& =t_{i+1}-t_{i} .
\end{aligned}
$$

Two HEV transmission routes were investigated in this study: (i) transmission due to direct contact between infected and naïve pigs; (ii) indirect transmission via an environmental reservoir of the virus in the pen. A Bayesian model similar to the one described in Andraud et al. [25] was used. Briefly, on each sampling interval $D_{i}=\left[t_{i}, t_{i+1}\right]$ of duration $d_{i}$, the probability for a susceptible pig $j$ housed in pen $k$ to escape infection is given by:

$$
p_{i}^{(k)}=\exp \left(-d_{i}\left(\beta_{w} \pi_{i}^{(k)}+\beta_{E}^{(w)} \frac{E_{k i}^{(w)}}{N}\right)\right),
$$

where $\pi_{i}^{(k)}$ represents the proportion of shedding pigs in the time interval $D_{i}$ located in pen $k, E_{k i}^{(w)}$ is the environmental pool of viral particles in time interval $D_{i}$ in the pen, $\beta_{w}$ is the within-pen transmission rate by direct contact and $\beta_{E}^{(w)}$ is the within-pen environmental transmission rate. For each pig $j$, the time interval in which the infection occurred was determined by estimating the latent period $\lambda_{j}$. Let $D_{I_{j}}=\left[t_{I_{j}}, t_{I_{j}+1}\right]$ denote the time interval during which the first positive fecal sample was detected in pig $j$. The contribution of contact animal $j$ in pen $k$ to the likelihood model, i.e. the probability for its first positive fecal sample to stand in the interval $D_{I_{j}}=\left[t_{I_{j}}, t_{I_{j}+1}\right]$ is:

$L^{(j)}\left(D_{I}, \pi_{w}, E \mid \beta_{w}, \beta_{E}^{(w)}, \lambda, \delta\right)=\left\{\prod_{i=1}^{I_{j}} p_{l-1}^{(k)}\left(1-p_{I_{j}}^{k}\right)\right\} \times f_{\text {Lat }}\left(\lambda_{j}, \alpha, s\right)$,

The probability of infection (given by the first term of the equation aforementioned) is weighted by the probability that the estimated latent period $\lambda_{j}$ is consistent with the data observed in inoculated animals. $f_{\text {Lat }}$ represents the prior distribution of the latent period based on the estimation of the latent period in inoculated animals. The global likelihood is given by:

$$
L\left(D_{I}, \pi_{w}, E \mid \beta_{w}, \beta_{E}^{(w)}, \lambda, \delta\right)=\prod_{j=1}^{N_{c}} L^{(j)}\left(D_{I}, \pi_{w}, E \mid \beta_{w}, \beta_{E}^{(w)}, \lambda, \delta\right),
$$

where $N_{c}$ is the total number of contact pigs.

The direct and indirect transmission rates $\beta_{w}$ and $\beta_{E}^{(w)}$ respectively, the latent period $\lambda_{j}$ for each contact animal and the HEV clearance rate were estimated by Bayesian inference using Monte Carlov Markov Chain. An informative prior distribution based on Andraud et al. [25] was used for the environmental clearance rate $\delta$, which was assumed to be normally distributed with mean 0.3 and standard deviation 0.075 . The prior distributions of transmission parameters were based on the results obtained by Andraud et al. [25]; they were constructed such that the expected value is equal to the posterior mean and $33 \%$ of the prior mass covers the $95 \%$ confidence interval for parameters derived from data obtained by Andraud et al. [25,42] (normal distribution $(-2,3)$ and $(-13.5,5)$ for $\beta_{w}$ and $\beta_{E}^{(w)}$ respectively). The prior distribution of the latent period in contact pigs was based on the distribution of the latent period in inoculated pigs (gamma distribution $\Gamma(26,2)$ ).

Parameter updating was performed sequentially by the Metropolis-Hastings algorithm. Three chains were run with random initial conditions, 110000 steps per chain, a burnin of 10000 steps and thinning parameter of 10 . Convergence was assessed by visual inspection and diagnostic tests (autocorrelation, Heidelberger, Gelman-Rubin diagnostics).

The whole model was performed using the R software [40].

\section{Results}

\section{HEV-only infection experiment}

In this trial, the average HEV latent period in inoculated animals lasted 6.9 days $(5.8 ; 7.9)$ and average infectious period lasted 9.7 days $(8.2 ; 11.2)$ (Table 1$)$ [25]. Direct transmission rate was estimated at $0.15(0.03 ; 0.31)$ pigs per day and indirect transmission rate was estimated at $2 \cdot 10^{-6} \mathrm{~g} / \mathrm{ge} /$ day $\left(1 \cdot 10^{-7} ; 7 \cdot 10^{-6}\right)$ (Table 1) [25]. HEV serology results on individual blood samples for HEV-only infected pigs are presented in Additional file 1 [25].

\section{HEV shedding and seroconversion in the context of HEV/PRRSV co-infection}

HEV infection data are presented in Figures 2 and 3 for quantitative RT-PCR on fecal samples and serological results respectively. In our trial, all inoculated animals were infected by HEV. None of the 2 negative-control pigs excreted HEV from day 3 to day 49. Inoculated and contact animals started to shed HEV between 9 and 18 dpi and between 25 and 32 dpi respectively. All exposed individuals shed HEV until the end of the trial (49 dpi) (Figure 2). At 
Table 1 Summary of the infectious dynamics parameters and comparison with data from the HEV-only infection experiment [25]

\begin{tabular}{lll}
\hline & HEV + PRRSV & HEV alone $[25]$ \\
\hline Latent period (days) & 13.4 & 7.1 \\
& $(8.6 ; 17.1)$ & $(3.2 ; 12.3)$ \\
Infectious period (days) & 48.6 & 9.7 \\
& $(27.9 ; 84.6)$ & $(8.2 ; 11.2)$ \\
Seroconversion period (days) & 43.1 & 26.3 \\
& $(35.7 ; 52.2)$ & $(23.5 ; 29.5)$ \\
Direct transmission (days $\left.{ }^{-1}\right) \beta_{w}$ & 0.70 & 0.15 \\
& $\left(1.2 \cdot 10^{-3} ; 3.67\right)$ & $(0.03 ; 0.31)$ \\
Indirect transmission (g/ge/d) $\beta_{E}^{W}$ & $6.6 \cdot 10^{-6}$ & $2.0 \cdot 10^{-6}$ \\
& $\left(1.4 \cdot 10^{-10} ; 1.3 \cdot 10^{-4}\right)$ & $\left(1.1 \cdot 10^{-7} ; 7.0 \cdot 10^{-6}\right)$ \\
\hline
\end{tabular}

$\beta_{w}$ is the direct transmission rate, defined as the mean number of newly infected pigs generated by a single infectious individual in a fully susceptible population per day. $\beta_{E}^{w}$ represents the within-pen transmission rates related to the environmental component, defined as the mean number of newly infected pigs per HEV genome equivalent per gram of feces in the environment (see text for more details). Numbers in brackets are the upper and lower limits of the $95 \%$ credibility interval.

the necropsy stage, 14 livers out of 18 were positive, the 4 negative livers being from contact pigs (Figure 2).

The detection of anti-HEV antibodies was performed on all groups of animals until 49 dpi (Figure 3). None of the negative controls showed anti-HEV antibody response. Only 4 inoculated animals out of 9 produced anti-HEV antibodies between 35 and 49 dpi, 3 in group 2 and one in group 3; none of the inoculated animals from group 1 seroconverted. Seven contact individuals out of 9 seroconverted between 42 and $49 \mathrm{dpi}$, two from groups 1 and 2 and all three contact animals from group 3 (Figure 3 ).

\section{PRRSV infection and seroconversion in the context of HEV/PRRSV co-infection}

All animals inoculated with PRRSV were viremic from the first sampling time $(7 \mathrm{dpi})$. The viremia of contact animals started between 7 and 42 dpi. One contact individual did not show any detectable PRRSV viremia during the experiment (Figure 4). Finally, all animals except 2 contact individuals were viremic for PRRSV before HEV shedding was detected.

Regarding clinical data (data not shown), inoculated and contact animals showed hyperthermia (rectal temperature $>40{ }^{\circ} \mathrm{C}$ ) between 1 and $14 \mathrm{dpi}$ and 14 and $28 \mathrm{dpi}$, respectively. Coinfected pigs necropsied at $49 \mathrm{dpi}$ did not show any macroscopic lesion possibly linked to hepatitis.

\section{Quantification of HEV infection dynamics parameters in the context of HEV/PRRSV co-infection}

Convergence of MCMC was assessed through visual inspection and conventional diagnostic tests. Heidelberger and Geweke diagnostics failed to reject the convergence hypothesis, which was also supported by the GelmanRubin test based on three independent chains with a potential scale reduction factor (PSRF) close to $1.0(\leq 1.02)$ (Additional file 2).

\section{HEV latent and infectious periods}

The duration of the latent period in pigs inoculated with HEV and PRRSV was fitted to a gamma distribution with shape parameter $\alpha=25.7(11.6 ; 180.4)$ and scale parameter $s=0.5(0.08 ; 1.1)$ leading to an estimated mean duration of the latent period of 12.9 days $(12.8 ; 14.4)$. In contact animals, individual distributions of latent periods (Additional

\begin{tabular}{|c|c|c|c|c|c|c|c|c|c|c|c|c|c|c|c|c|c|c|c|c|c|}
\hline & $\mathrm{dpi}$ & 2 & & 7 & 9 & 11 & 14 & 16 & 18 & 21 & 23 & 25 & 28 & 32 & 35 & 39 & 42 & 44 & 46 & 49 & $\begin{array}{c}\text { Necropsy } \\
\text { (liver) }\end{array}$ \\
\hline & Control & 0 & 0 & 0 & 0 & 0 & 0 & 0 & 0 & 0 & 0 & 0 & 0 & 0 & 0 & 0 & 0 & 0 & 0 & 0 & 0 \\
\hline & Control & 0 & 0 & 0 & 0 & 0 & 0 & 0 & 0 & 0 & 0 & 0 & 0 & 0 & 0 & 0 & 0 & 0 & 0 & 0 & 0 \\
\hline \multirow[t]{6}{*}{ Group 1} & Inoculated & 0 & 0 & 0 & $1.08 .10^{5}$ & $0^{*}$ & $2.23 .10^{4}$ & $2.23 .10^{5}$ & $1.24 .10^{6}$ & $2.62 .10^{6}$ & $1.38 .10^{7}$ & $1.35 .10^{7}$ & $5.96 .10^{6}$ & $8.27 .10^{6}$ & $7.51 .10^{6}$ & $2.95 .10^{7}$ & $4.43 .10^{7}$ & $8.02 .10^{6}$ & $8.76 .10^{5}$ & $1.49 \cdot 10^{7}$ & $1.46 .10^{6}$ \\
\hline & Inoculated & 0 & 0 & 0 & 0 & 0 & $2.23 .10^{4}$ & $1.72 .10^{5}$ & $1.91 .10^{6}$ & $6.80 .10^{6}$ & $2.24 .10^{7}$ & $3.56 .10^{7}$ & $7.66 .10^{7}$ & $1.32 .10^{8}$ & $9.91 .10^{7}$ & $6.76 .10^{7}$ & $1.51 .10^{7}$ & $1.09 .10^{8}$ & $4.01 \cdot 10^{7}$ & $5.85 .10^{7}$ & $1.87 .10^{6}$ \\
\hline & Inoculated & 0 & 0 & 0 & 0 & 0 & 0 & 0 & $1.50 .10^{5}$ & $1.53 .10^{6}$ & $5.85 .10^{5}$ & $1.14 .10^{7}$ & $1.30 .10^{7}$ & $4.06 .10^{7}$ & $3.99 .10^{7}$ & $9.27 \cdot 10^{7}$ & $4 \cdot 28 \cdot 10^{7}$ & $5.02 .10^{6}$ & $7.20 .10^{7}$ & $6.40 .10^{7}$ & $1.02 .10^{6}$ \\
\hline & Contact & 0 & 0 & 0 & 0 & 0 & 0 & 0 & 0 & 0 & 0 & $2.67 .10^{5}$ & $1.16 .10^{6}$ & $1.79 .10^{7}$ & 1.54.10 & $3.75 .10^{7}$ & $1.92 .10^{7}$ & $1.42 .10^{7}$ & $5.31 .10^{7}$ & $4.87 .10^{7}$ & $8.72 .10^{5}$ \\
\hline & Contact & 0 & 0 & 0 & 0 & 0 & 0 & 0 & 0 & 0 & 0 & 0 & $2.91 .10^{5}$ & $3.68 .10^{6}$ & $3.51 .10^{6}$ & $6.24 .10^{5}$ & $1.53 .10^{5}$ & $4.91 .10^{5}$ & $1.17 .10^{6}$ & $3.49 .10^{5}$ & 0 \\
\hline & Contact & 0 & 0 & 0 & 0 & 0 & 0 & 0 & 0 & 0 & 0 & $1.78 .10^{2}$ & $8.84 .10^{5}$ & $1.43 .10^{6}$ & $3.04 .10^{6}$ & $5.43 .10^{5}$ & $1.20 .10^{6}$ & $4.29 .10^{6}$ & $1.91 .10^{6}$ & $1.20 .10^{6}$ & 0 \\
\hline \multirow[t]{6}{*}{ Group 2} & Inoculated & 0 & 0 & 0 & 0 & 0 & 0 & $1.56 .10^{5}$ & $1.09 .10^{6}$ & $1.57 .10^{6}$ & $2.81 .10^{6}$ & $1.67 .10^{7}$ & $2.69 .10^{7}$ & $8.71 .10^{7}$ & $6.33 \cdot 10^{7}$ & $4.44 .10^{6}$ & $3.92 .10^{6}$ & $1.64 .10^{7}$ & $9.92 .10^{5}$ & $1.45 .10^{5}$ & 1.56.10 \\
\hline & Inoculated & 0 & 0 & 0 & 0 & 0 & 0 & $2.19 .10^{5}$ & $5.44 .10^{5}$ & $6.85 .10^{6}$ & $1.86 .10^{7}$ & $1.51 .10^{7}$ & $2.31 .10^{7}$ & $1.55 .10^{7}$ & $1.79 .10^{7}$ & $2 \cdot 57.10^{7}$ & $7.88 .10^{6}$ & $1.37 .10^{8}$ & $1.97 .10^{7}$ & $6.80 \cdot 10^{6}$ & $2.42 .10^{6}$ \\
\hline & Inoculated & 0 & 0 & 0 & 0 & 0 & $1.03 .10^{5}$ & $6.97 .10^{5}$ & $5.05 .10^{8}$ & $3.85 .10^{7}$ & $4.20 .10^{7}$ & 3.96.10 & $6.72 .10^{7}$ & $1.25 .10^{8}$ & $1.39 .10^{8}$ & $5.93 .10^{7}$ & $8 \cdot 10 \cdot 10^{7}$ & $1.32 .10^{8}$ & $1.13 .10^{7}$ & $4.86 .10^{6}$ & $3.59 .10^{5}$ \\
\hline & Contact & 0 & 0 & 0 & 0 & 0 & 0 & 0 & 0 & 0 & 0 & $1.65 .10^{6}$ & $0^{*}$ & $1.45 .10^{6}$ & $4.12 .10^{6}$ & $4.94 .10^{6}$ & abs & $1.18 .10^{7}$ & $2.51 .10^{7}$ & $5.55 .10^{7}$ & $1.12 .10^{6}$ \\
\hline & Contact & 0 & 0 & 0 & 0 & 0 & 0 & 0 & 0 & 0 & 0 & 0 & 0 & $1.82 .10^{6}$ & $5.38 .10^{5}$ & $8.40 .10^{6}$ & $4.43 .10^{6}$ & $8.37 .10^{7}$ & $1.43 .10^{7}$ & $5.24 .10^{6}$ & $1.69 .10^{6}$ \\
\hline & Contact & 0 & 0 & 0 & 0 & 0 & 0 & 0 & 0 & 0 & 0 & 0 & 0 & $1.47 .10^{6}$ & $7.26 .10^{5}$ & $1.97 .10^{7}$ & $3.03 \cdot 10^{7}$ & $4.37 .10^{7}$ & $2.49 .10^{8}$ & $3.07 .10^{8}$ & $3.44 .10^{6}$ \\
\hline \multirow[t]{6}{*}{ Group 3} & Inoculated & 0 & 0 & 0 & 0 & 0 & 0 & $2.37 .10^{5}$ & $3.12 .10^{5}$ & $3.88 .10^{6}$ & $6.75 .10^{5}$ & $7.98 .10^{6}$ & $1.05 .10^{7}$ & $4.21 .10^{7}$ & $2.64 .10^{7}$ & $4.26 \cdot 10^{7}$ & $1.51 .10^{7}$ & $2.90 .10^{7}$ & $1.60 .10^{7}$ & $2.08 .10^{8}$ & $3.24 .10^{6}$ \\
\hline & Inoculated & 0 & 0 & 0 & 0 & 0 & 0 & 0 & $6.06 .10^{5}$ & $9.54 .10^{5}$ & $4.08 .10^{5}$ & $3.22 .10^{6}$ & 1.55.107 & $3.02 .10^{7}$ & 1.76.10 & $1.80 \cdot 10^{7}$ & 1.88.10 & $8.52 .10^{7}$ & 1.85.10 & $5.75 .10^{7}$ & $2.04 .10^{4}$ \\
\hline & Inoculated & 0 & 0 & 0 & 0 & 0 & $8.04 .10^{4}$ & 4.35.10 & $2.26 .10^{6}$ & $1.14 .10^{7}$ & $2.25 .10^{7}$ & $1.73 .10^{7}$ & $2.37 .10^{7}$ & $3.66 .10^{7}$ & $3.17 .10^{7}$ & $1.04 .10^{7}$ & $4.39 .10^{6}$ & $4.09 .10^{7}$ & $1.43 .10^{7}$ & $5.61 .10^{7}$ & $9.63 .10^{5}$ \\
\hline & Contact & 0 & 0 & 0 & 0 & 0 & 0 & 0 & 0 & 0 & 0 & 0 & 0 & $8.80 .10^{4}$ & $2.04 .10^{5}$ & $2.34 .10^{6}$ & $3.40 .10^{5}$ & $4.04 .10^{5}$ & $5.02 .10^{5}$ & $7.39 .10^{5}$ & 0 \\
\hline & Contact & 0 & 0 & 0 & 0 & 0 & 0 & 0 & 0 & 0 & 0 & 0 & 0 & $8.26 .10^{6}$ & $1.50 .10^{7}$ & $8.47 .10^{7}$ & $0^{*}$ & $1.45 .10^{7}$ & $8.67 .10^{7}$ & $1.03 .10^{8}$ & $5.87 .10^{5}$ \\
\hline & Contact & 0 & 0 & 0 & 0 & 0 & 0 & 0 & 0 & 0 & 0 & 0 & 0 & $1.31 .10^{6}$ & $8.42 .10^{5}$ & $1.12 .10^{6}$ & $0^{*}$ & $4.41 .10^{4}$ & $7.78 .10^{5}$ & $8.29 .10^{5}$ & 0 \\
\hline \multicolumn{22}{|c|}{$\begin{array}{l}\text { Figure } 2 \text { HEV RNA quantification in fecal and liver samples from HEV/PRRSV co-infected animals and contact pigs. Quantitative HEV RT- } \\
\text { PCR results on individual fecal samples (HEV copies/g of feces) at each sampling time and from liver samples at necropsy. Shaded zones } \\
\text { correspond to periods in which infected individuals were considered infectious, corresponding to the time between the first and last HEV } \\
\text { positive fecal samples for each animal. dpi: day post infection; *tested in duplicate; abs: missing. }\end{array}$} \\
\hline
\end{tabular}




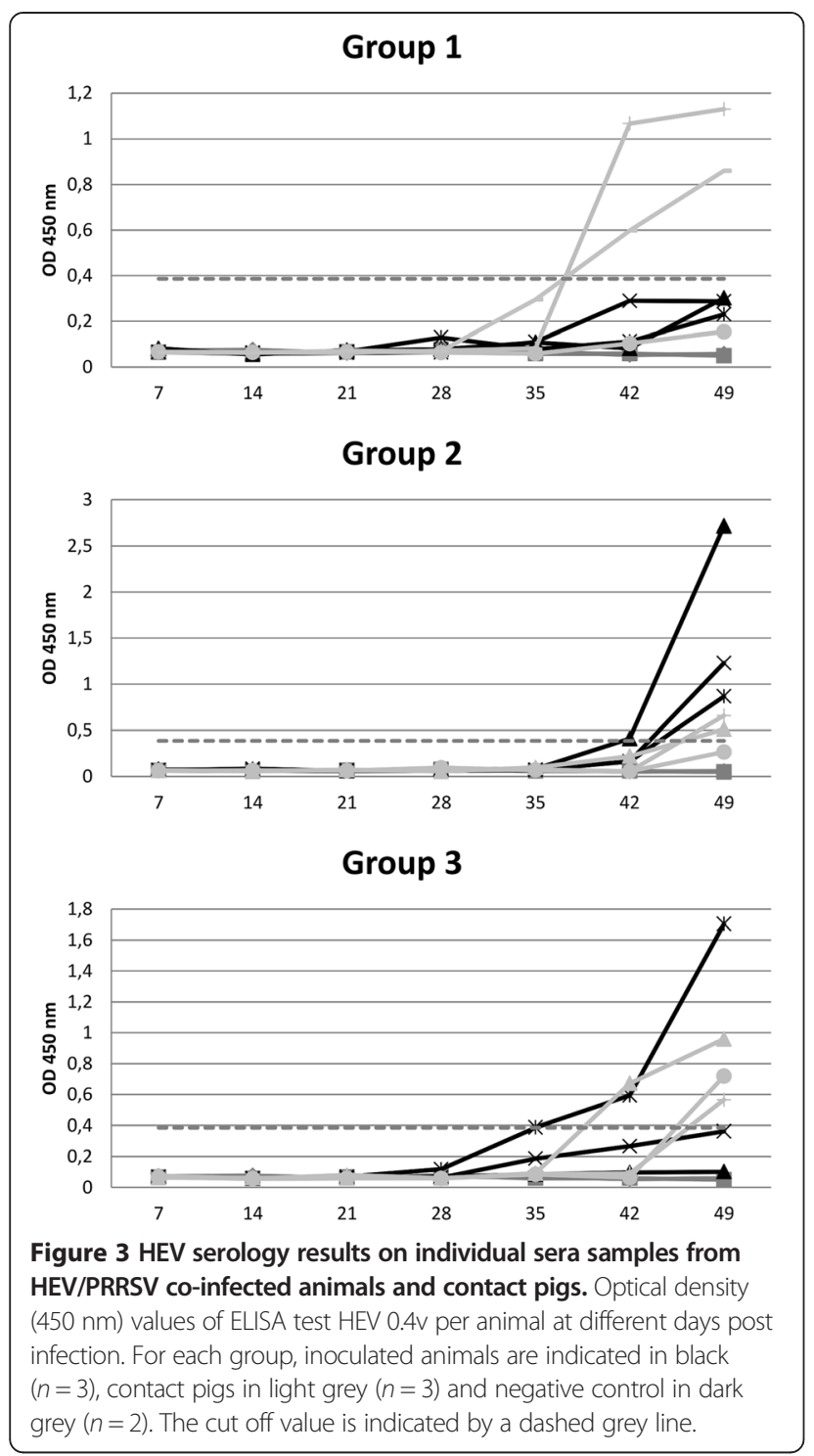

file 2) were merged to obtain a global distribution of the latent period, leading to a mean latent period duration of 13.4 days $(8.6 ; 17.1)$ (Table 1$)$.

The duration of the infectious period was fitted to a lognormal distribution, leading to an estimated mean duration of the infectious period of 48.6 days $(27.9 ; 84.6)$ (Table 1$)$.

\section{Estimation of time to HEV seroconversion}

Time-to HEV seroconversion was fitted to log-normal distribution, with means 43.1 days $(35.7 ; 52.2)$ with PRRSV co-infection and 26.3 days $(23.5 ; 29.5)$ with only HEV infection (Table 1). The duration of the infectious period was significantly associated with the earliness of the humoral immune response. An absent or late immune response was related to a lengthening of the infectious period duration showed by a delay in time-to end of shedding (Hazard Ratio HR $=0.35(0.19 ; 0.64)$ ) (Figure 5).

\section{HEV shedding and accumulation in the environment}

The distribution of the HEV shed viral load with time (with and without co-infection) is shown in Figure 6. PRRSV infection was found to be significantly associated with the increase of the quantity of HEV particles shed by inoculated animals $(P=0.05)$ from the linear mixed model accounting for repeated measurements. The interaction between time and PRRSV infection was also significant and positive, i.e. the impact of the PRRSV infection increased with time $(P=0.04)$. However, the effect of the PRRSV infection was not found to be statistically significant in contact animals $(P>0.05)$.

The viral load accumulated in the environment was modeled for each experimental pen (Figure 7). The environment was HEV-free until 15 to $20 \mathrm{dpi}$; then the environmental load increased and reached $1.0 .10^{8}$ to $1.5 \cdot 10^{8} \mathrm{ge} / \mathrm{g}$ of feces until the end of the trial.

\section{HEV transmission parameters}

The results show that, in experimental conditions, one infectious pig was able to infect 0.70 pig per day by direct contact $\left(\beta_{w}=0.70\left(1.18 \cdot 10^{-3} ; 3.67\right)\right)$ (Table 1). The indirect transmission rate can be considered as the average number of animals that can be infected by a single genome equivalent present in the pen environment $\left(\beta_{E}^{(w)}=6.59 \cdot 10^{-6} \mathrm{~g} / \mathrm{ge} /\right.$ day $\left.\left(1.43 \cdot 10^{-10} ; 1.27 \cdot 10^{-4}\right)\right)$. In other words, the inverse of $\beta_{E}^{(w)}$ corresponds to the average number of viral copy number of genome per gram of feces in the environmental pool required to infect one animal in one day, i.e. $1.51 \cdot 10^{5} \mathrm{ge} / \mathrm{g} /$ day $\left(7.86 \cdot 10^{3} ; 7.00 \cdot 10^{9}\right)$ (Table 1$)$.

\section{Discussion}

Several studies suggested a possible link between HEV and PRRSV infections [30-32]. Our study was aimed at evaluating the impact of PRRSV infection on hepatitis $\mathrm{E}$ dynamics of infection through an experimental HEV/ PRRSV co-infection trial. As shown in Table 1, the comparison of the results with those derived from a previous infection trial with HEV alone [25] evidenced a modification of hepatitis $\mathrm{E}$ infection dynamics in the presence of PRRSV. Although the two trials were not carried out simultaneously, they were conducted under the same experimental conditions making the comparison of the results fully relevant (same experimental facilities, same handlers, pigs from the same SPF herd and genetically similar, same age of the animals, same sex ratio, same HEV strain, same dose, same inoculation protocol and same contact structure).

HEV shedding was delayed in case of PRRSV co-infection, with a latent period estimated to 13.4 days, against 7.1 days with HEV alone [25], i.e. an increase by a factor of 1.9. In the Bouwknegt et al. trial, the latent period was estimated at only 3 days in intravenously inoculated animals [20], confirming that the route of inoculation 


\begin{tabular}{|c|c|c|c|c|c|c|c|c|}
\hline \multirow{8}{*}{ Group 1} & $\mathrm{dpi}$ & 7 & 14 & 21 & 28 & 35 & 42 & 49 \\
\hline & Control & $n t$ & $\mathrm{nt}$ & N/A & $n t$ & N/A & N/A & $\mathrm{nt}$ \\
\hline & Control & $n t$ & $\mathrm{nt}$ & $\mathrm{nt}$ & $\mathrm{N} / \mathrm{A}$ & $\mathrm{N} / \mathrm{A}$ & $N / A$ & $N / A$ \\
\hline & Inoculated & 28.94 & 26.39 & 35.52 & 33.43 & 28.08 & $N / A$ & $N / A$ \\
\hline & Inoculated & 30.74 & 29.27 & 33.43 & 39.25 & N/A & N/A & N/A \\
\hline & Inoculated & 32.30 & 29.88 & 33.30 & 38.68 & 35.92 & $N / A$ & $N / A$ \\
\hline & Contact & 31.76 & 29.16 & 29.64 & 28.96 & 37.73 & $\mathrm{~N} / \mathrm{A}$ & $N / A$ \\
\hline & Contact & N/A & 30.19 & 35.32 & 29.44 & $N / A$ & N/A & $N / A$ \\
\hline \multirow{4}{*}{ Group 2} & Contact & N/A & N/A & N/A & N/A & N/A & 31.39 & 22.26 \\
\hline & Inoculated & 28.26 & 28.19 & 31.27 & 27.42 & N/A & $N / A$ & $N / A$ \\
\hline & Inoculated & 28.36 & 30.33 & 33.20 & 26.4 & 29.4 & $N / A$ & $N / A$ \\
\hline & Inoculated & 28.69 & 30.37 & 35.00 & 26.61 & N/A & N/A & N/A \\
\hline \multirow{9}{*}{ Group 3} & Contact & 35.11 & 28.68 & 31.97 & 24.27 & 31.2 & 26.03 & $N / A$ \\
\hline & Contact & $\mathrm{N} / \mathrm{A}$ & $\mathrm{N} / \mathrm{A}$ & 36.69 & 25.4 & 44.63 & $N / A$ & $N / A$ \\
\hline & Contact & 36.95 & $\mathrm{~N} / \mathrm{A}$ & 33.13 & 24.37 & 29.28 & N/A & $\mathrm{N} / \mathrm{A}$ \\
\hline & Inoculated & 29.12 & 26.03 & 32.21 & 30.89 & N/A & N/A & $N / A$ \\
\hline & Inoculated & 30.49 & 26.82 & 32.39 & 27.37 & N/A & 36.54 & N/A \\
\hline & Inoculated & 29.04 & 38.31 & 43.52 & $\mathrm{~N} / \mathrm{A}$ & $\mathrm{N} / \mathrm{A}$ & $\mathrm{N} / \mathrm{A}$ & $N / A$ \\
\hline & Contact & N/A & 32.15 & 29.99 & 25.74 & $\mathrm{~N} / \mathrm{A}$ & 28.43 & $\mathrm{~N} / \mathrm{A}$ \\
\hline & Contact & N/A & 34.22 & 33.05 & 36.73 & 27.66 & $N / A$ & N/A \\
\hline & Contact & N/A & N/A & N/A & N/A & N/A & N/A & N/A \\
\hline
\end{tabular}

modifies viral fate. The infectious period was longer with PRRSV co-infection: 48.6 days, against 9.7 days with HEV alone, i.e. an increase by a factor of $5(p<0.01)$. These results were therefore closer to estimates obtained from field data $(27$ days $(20 ; 39))$ than experimental results obtained with HEV only [26]). In the trial described by Bouwknegt et al., the infectious period was estimated between 13 and 49 days according to replications, showing a high inter-individual variability [20]. Moreover, the origin of the animals included in this study and their status regarding PRRSV were not mentioned.

HEV shedding in inoculated individuals was also significantly increased with PRRSV/HEV co-infection. However, the effect of PRRSV infection on the quantity of shed viral particles was not significant in contact animals. This could

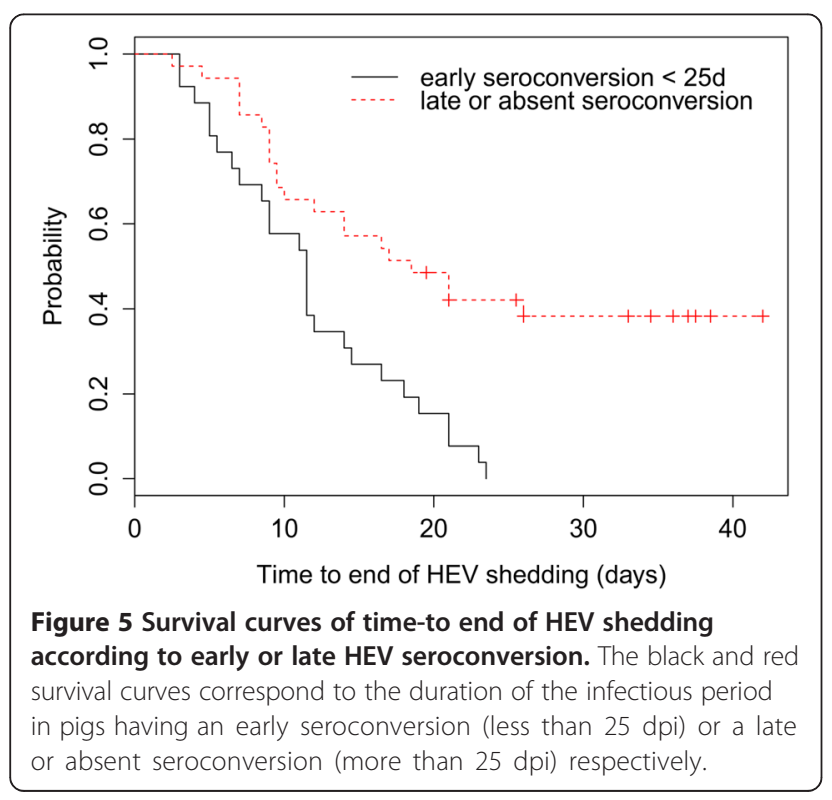

be explained by the low number of animals included - especially since one contact animals was lately infected by PRRSV and another did not show any PRRSV viremia during the experiment - and by a large inter-individual variability in contact animals. As a consequence of the longer shedding period and the higher quantity of viral particles shed in feces of co-infected animals, the viral load accumulated in the environment was higher with PRRSV coinfection with more than $10^{8} \mathrm{HEV}$ ge/g of feces estimated in the environment, which causes a higher and longer infection pressure on susceptible animals. The direct transmission rate when animals were co-infected was increased by a factor of 4.7 ( 0.70 versus 0.15 per day with HEV infection only [25]). Thus the direct transmission route played a more important role in HEV transmission when animals

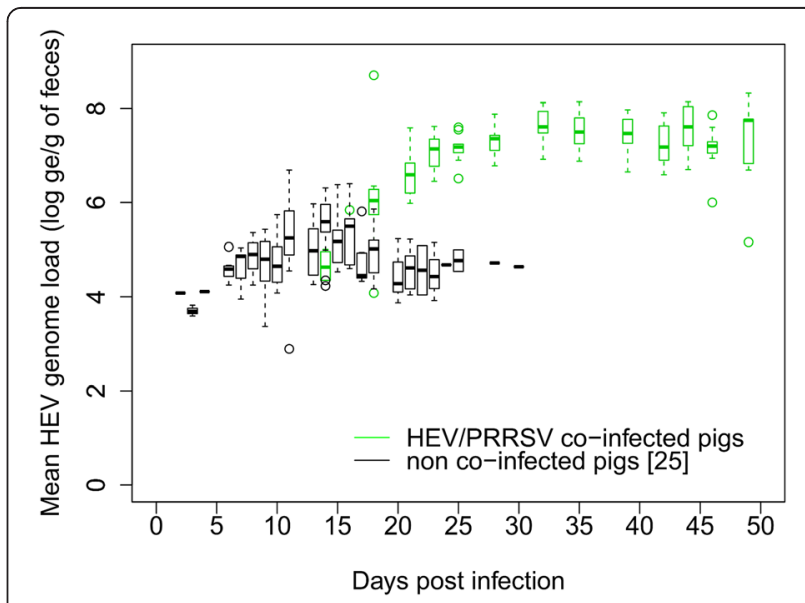

Figure 6 Distribution of the number of HEV genome equivalent (log ge/g feces) shed by individual pigs with time in inoculated animals with or without PRRSV co-infection. Co-infected animals are indicated in green $(n=9)$, only-HEV infected animals [25] are in black $(n=18)$. 


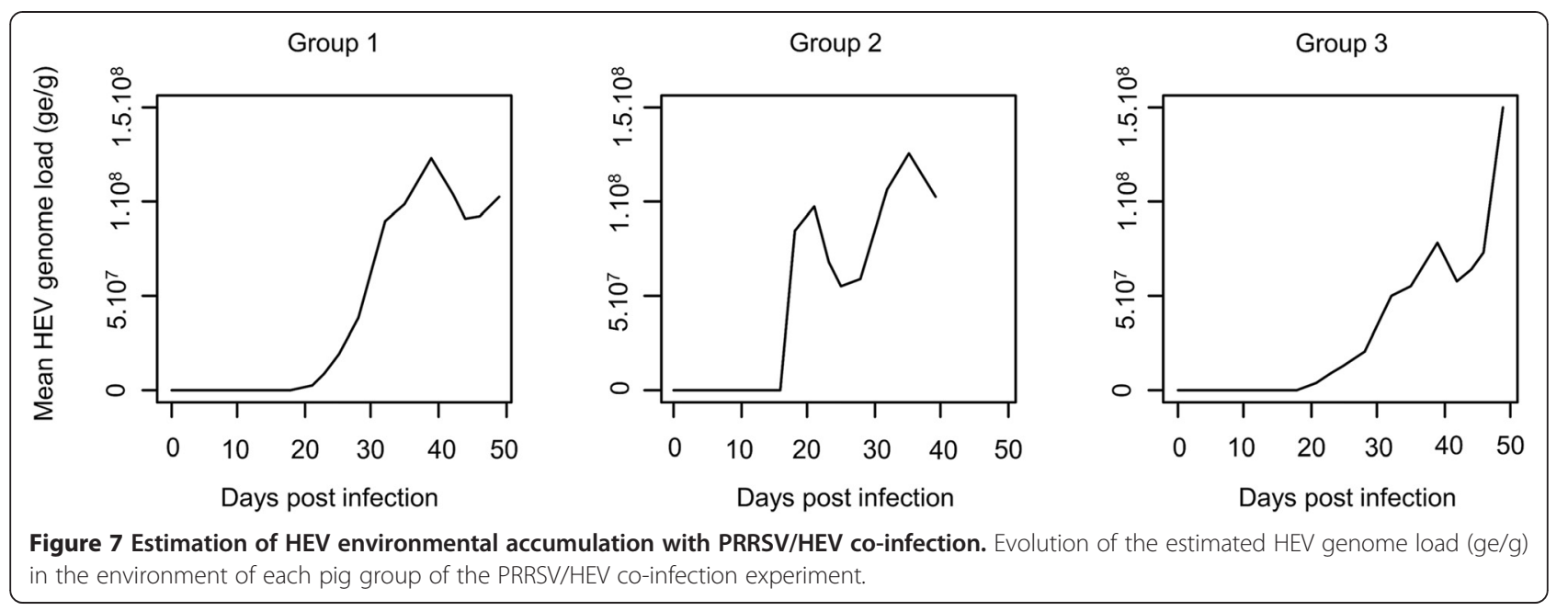

were co-infected which was consistent with the larger amount of HEV particles shed individually than in HEV infected pigs only. The indirect transmission rate was 3.3 times higher with co-infection $\left(6.6 \cdot 10^{-6}\right.$ and $2.0 \cdot 10^{-6} \mathrm{~g} / \mathrm{ge} /$ day respectively [25]). Otherwise stated, 3.3 times less viral particles were required to infect a co-infected animal $\left(1.5 \cdot 10^{5}\right.$ versus $5.0 \cdot 10^{5}$ ge/g for HEV only infected piglets [25]). Because inoculated and contact animals (except two contact pigs) were infected by PRRSV before HEV shedding, these data suggest a higher HEV susceptibility in PRRSV co-infected pigs. In a model built from an experimental HEV infection by intravenous route, Bouwknegt et al. showed that the HEV oral dose for which the infection probability was equal to $50 \%$ would be $1.4 \cdot 10^{6}$ ge/g [22], which was 10 times more than the dose required to infect a PRRSV co-infected pig in our study. These data are consistent with the hypothesis of a higher HEV infection susceptibility in PRRSV co-infected pigs.

The time-to HEV seroconversion was 1.6 times longer in PRRSV co-infected pigs than in HEV only infected pigs (43.1 and 26.3 days respectively [25]). This impaired immune response was significantly associated with a lengthening of the infectious period duration and could thus explain the presence of viral particles in livers when pigs were euthanized more than 49 days post infection for the inoculated ones. However, this study did not aim at investigating the mechanisms leading to a possible immune failure linked to PRRSV infection and the mechanisms causing a chronic HEV infection. In humans, immunopathogenic mechanisms leading to chronic hepatitis $\mathrm{E}$ are poorly known. The role of cellular immunity in chronic hepatitis $\mathrm{E}$ control has been shown [3,35,36]. A study was led on patients suffering from HIV and chronically infected with HEV [34]. One of them had a low anti-HEV lymphocyte $\mathrm{T} \mathrm{CD} 4+$ rate, a persistent viremia (longer than 24 months) and a delayed anti-HEV seroconversion. Thus, though immune mechanisms still need to be clarified, literature data suggest that an impaired innate and adaptive immune response could lead to chronic HEV infection in humans. In pigs, the immunopathogenic mechanisms linked to PRRSV infection are not fully understood yet, but PRRSV infection clearly results in a late adaptive immune response [43,44]. Thus the delayed anti-HEV seroconversion and the lengthening of the infectious period duration that we observed in PRRSV co-infected pigs seem consistent with the immunopathogenic mechanisms of chronic hepatitis $\mathrm{E}$ that have been described in humans (impaired cellular and humoral immune response) and could be explained by a specific orientation of the immune response linked to PRRSV infection. The increase of the duration of the latent period might be explained by the activation of the innate immune response linked to the PRRSV infection, delaying HEV shedding but this would require further work to assess the underlying mechanisms.

To our knowledge, this work is the first study focusing on the impact of HEV/PRRSV co-infection on hepatitis E epidemiology in pigs. These results show that PRRSV has a major impact on HEV infection dynamics and that HEV/PRRSV co-infection could lead to extended HEV shedding and maybe chronic infection. This chronicity may dramatically increase the risk of pig livers containing HEV at slaughter age. Immunopathogenic mechanisms leading to a chronic HEV infection have to be further investigated. This study shows an important interaction between an animal health concern - PRRSV, which dramatically affects the competitiveness of pig farms, and a zoonotic pathogen - HEV, which has a major impact in human health. These data emphasize the necessity to manage human and animal health globally and the importance of PRRSV eradication programs, which could be a major lever in the control of hepatitis E. 


\section{Additional files}

Additional file 1: HEV serology results on individual sera samples for only-HEV infected pigs [25]. Optical density $(450 \mathrm{~nm})$ values of ELISA test HEV $0.4 \mathrm{~V}$ per animal at different day post infection. Shaded zones correspond to the period in which individuals were considered HEV seropositive. dpi: days post infection, abs: missing.

\section{Additional file 2: Estimation of transmission parameters by} Bayesian inference (MCMC estimation, 3 chains, 110000 iterations, 10000 burnin iterations, thinning interval $=10$ ). $\beta_{w}$ is the direct transmission rate, defined as the mean number of newly infected pigs generated by a single infectious individual in a fully susceptible population per day. $\beta_{E}^{W}$ represents the within-pen transmission rates related to the environmental component, defined as the mean number of newly infected pigs per viral particle per gram of feces in the environment. $\delta$ is the HEV clearance rate, taking into account feces elimination through the metallic flat deck and HEV destruction in the environment. $\lambda_{1}$ to $\lambda_{9}$ are latent periods for contact animals (see text for more details).

\section{Competing interests}

The authors declare that they have no competing interests.

\section{Authors' contributions}

MS analyzed the data, participated to the sample analyses and drafted the manuscript, EB analyzed the HEV samples and interpreted the results, MA developed the mathematical model and participated to the data analyses, FE participated in the animal experiment, monitored and pre-treated the samples and participated to the analysis of PRRSV samples, PR and OB analyzed and interpreted the results from PRSSV samples, NP supervised the HEV-related laboratory work and participated in the coordination of the study, NR conceived, coordinated the study and participated in the animal experiment and the data analyses. All co-authors revised the manuscript and approved the final submitted version.

\section{Acknowledgements}

This study was funded by the French Research Agency (ANR) as part of the research project called "HEVECODYN" and by the European Community's Seventh Framework Program under the project PREDEMICS (grant agreement number 278433). We thank Yann Bailly and Frédéric Paboeuf for their excellent technical management of the experiment.

\section{Author details}

${ }^{1}$ ANSES, Laboratoire de Ploufragan-Plouzané, BP 53, 22440 Ploufragan, France. Université européenne de Bretagne, 35000 Rennes, France. ${ }^{3}$ UMR 1161 Virology, ANSES, Laboratoire de Santé Animale, 94706 Maisons-Alfort, France. 4UMR 1161 Virology, INRA, 94706 Maisons-Alfort, France. ${ }^{5}$ UMR 1161 Virology, Paris Est University, École Nationale Vétérinaire d'Alfort, 94706 Maisons-Alfort, France.

\section{Received: 20 January 2015 Accepted: 11 May 2015}

\section{Published online: 06 June 2015}

\section{References}

1. Emerson SU, Purcell RH (2003) Hepatitis E virus. Rev Med Virol 13:145-154

2. Gerolami R, Moal V, Colson P (2008) Chronic hepatitis E with cirrhosis in a kidney-transplant recipient. N Engl J Med 358:859-860

3. Kamar N, Garrouste C, Haagsma EB, Garrigue V, Pischke S, Chauvet C, Dumortier J, Cannesson A, Cassuto-Viguier E, Thervet E, Conti F, Lebray P, Dalton HR, Santella R, Kanaan N, Essig M, Mousson C, Radenne S, Roque-Afonso AM, Izopet J, Rostaing L (2011) Factors associated with chronic hepatitis in patients with hepatitis $E$ virus infection who have received solid organ transplants. Gastroenterology 140:1481-1489

4. Aggarwal R, Naik S (2009) Epidemiology of hepatitis E: current status. J Gastroenterol Hepatol 24:1484-1493

5. Balayan MS (1997) Epidemiology of hepatitis E virus infection. J Viral Hepat 4:155-165

6. Purcell RH, Emerson SU (2008) Hepatitis E: an emerging awareness of an old disease. J Hepatol 48:494-503
7. Dalton HR, Bendall R, ljaz S, Banks M (2008) Hepatitis E: an emerging infection in developed countries. Lancet Infect Dis 8:698-709

8. Rose N, Lunazzi A, Dorenlor V, Merbah T, Eono F, Eloit M, Madec F, Pavio N (2011) High prevalence of Hepatitis E virus in French domestic pigs. Comp Immunol Microbiol Infect Dis 34:419-427

9. Bouquet J, Tesse S, Lunazzi A, Eloit M, Rose N, Nicand E, Pavio N (2011) Close similarity between sequences of hepatitis $E$ virus recovered from humans and swine, France, 2008-2009. Emerg Infect Dis 17:2018-2025

10. Meng XJ, Halbur PG, Shapiro MS, Govindarajan S, Bruna JD, Mushahwar IK, Purcell RH, Emerson SU (1998) Genetic and experimental evidence for cross-species infection by swine hepatitis E virus. J Virol 72:9714-9721

11. van der Poel WH, Verschoor F, van der Heide R, Herrera MI, Vivo A, Kooreman M, de Roda Husman AM (2001) Hepatitis E virus sequences in swine related to sequences in humans, The Netherlands. Emerg Infect Dis 7:970-976

12. Colson P, Borentain P, Queyriaux B, Kaba M, Moal V, Gallian P, Heyries L, Raoult D, Gerolami R (2010) Pig liver sausage as a source of hepatitis E virus transmission to humans. J Infect Dis 202:825-834

13. Colson P, Romanet P, Moal V, Borentain P, Purgus R, Benezech A, Motte A, Gerolami R (2012) Autochthonous infections with hepatitis E virus genotype 4, France. Emerg Infect Dis 18:1361-1364

14. Deest G, Zehner L, Nicand E, Gaudy-Graffin C, Goudeau A, Bacq Y (2007) Autochthonous hepatitis $\mathrm{E}$ in France and consumption of raw pig meat. Gastroenterol Clin Biol 31:1095-1097 (in French)

15. Moal V, Gerolami R, Colson P (2012) First human case of co-infection with two different subtypes of hepatitis $E$ virus. Intervirology 55:484-487

16. Motte A, Roquelaure B, Galambrun C, Bernard F, Zandotti C, Colson P (2012) Hepatitis $E$ in three immunocompromized children in southeastern France. J Clin Virol 53:162-166

17. Pavio N, Meng XJ, Renou C (2010) Zoonotic hepatitis E: animal reservoirs and emerging risks. Vet Res 41:46

18. Lewis HC, Wichmann O, Duizer E (2010) Transmission routes and risk factors for autochthonous hepatitis $E$ virus infection in Europe: a systematic review. Epidemiol Infect 138:145-166

19. Balayan MS, Usmanov RK, Zamyatina NA, Djumalieva DI, Karas FR (1990) Brief report: experimental hepatitis E infection in domestic pigs. J Med Virol 32:58-59

20. Bouwknegt M, Frankena K, Rutjes SA, Wellenberg GJ, de Roda Husman AM, van der Poel WH, de Jong MC (2008) Estimation of hepatitis E virus transmission among pigs due to contact-exposure. Vet Res 39:40

21. Bouwknegt M, Rutjes SA, Reusken CB, Stockhofe-Zurwieden N, Frankena K, de Jong MC, de Roda Husman AM, Poel WH (2009) The course of hepatitis E virus infection in pigs after contact-infection and intravenous inoculation. BMC Vet Res 5:7

22. Bouwknegt M, Teunis PF, Frankena $K$, de Jong MC, de Roda Husman AM (2011) Estimation of the likelihood of fecal-oral HEV transmission among pigs. Risk Anal 31:940-950

23. Casas M, Pina S, de Deus N, Peralta B, Martin M, Segales J (2009) Pigs orally inoculated with swine hepatitis $E$ virus are able to infect contact sentinels. Vet Microbiol 138:78-84

24. Kasorndorkbua C, Thacker BJ, Halbur PG, Guenette DK, Buitenwerf RM, Royer RL, Meng XJ (2003) Experimental infection of pregnant gilts with swine hepatitis E virus. Can J Vet Res 67:303-306

25. Andraud M, Dumarest M, Cariolet R, Aylaj B, Barnaud E, Eono F, Pavio N, Rose N (2013) Direct contact and environmental contaminations are responsible for HEV transmission in pigs. Vet Res 44:102

26. Backer JA, Berto A, McCreary C, Martelli F, van der Poel WHM (2012) Transmission dynamics of hepatitis $E$ virus in pigs: Estimation from field data and effect of vaccination. Epidemics 4:86-92

27. Casas M, Cortes R, Pina S, Peralta B, Allepuz A, Cortey M, Casal J, Martin M (2011) Longitudinal study of hepatitis E virus infection in Spanish farrow-tofinish swine herds. Vet Microbiol 148:27-34

28. de Deus N, Casas M, Peralta B, Nofrarias M, Pina S, Martin M, Segalés J (2008) Hepatitis E virus infection dynamics and organic distribution in naturally infected pigs in a farrow-to-finish farm. Vet Microbiol 132:19-28

29. Rose N, Pavio N (2014) Epidémiologie du virus de l'hépatite E chez le porc: comment limiter l'exposition des consommateurs. Journées Recherche Porcine 46:159-168 (in French)

30. de Deus N, Seminati C, Pina S, Mateu E, Martin M, Segalés J (2007) Detection of hepatitis $E$ virus in liver, mesenteric lymph node, serum, bile and faeces of naturally infected pigs affected by different pathological conditions. Vet Microbiol 119:105-114 
31. Martelli F, Toma S, Di Bartolo I, Caprioli A, Ruggeri FM, Lelli D, Bonci M, Ostanello F (2010) Detection of Hepatitis E Virus (HEV) in Italian pigs displaying different pathological lesions. Res Vet Sci 88:492-496

32. Mao J, Zhao Y, She R, Xiao P, Tian J, Chen J (2013) One case of swine hepatitis $E$ virus and porcine reproductive and respiratory syndrome virus co-infection in weaned pigs. Virol J 10:341

33. Kamar N, Selves J, Mansuy JM, Ouezzani L, Peron JM, Guitard J, Cointault O, Esposito L, Abravanel F, Danjoux M, Durand D, Vinel JP, Izopet J, Rostaing L (2008) Hepatitis E virus and chronic hepatitis in organ-transplant recipients. N Engl J Med 358:811-817

34. Kenfak-Foguena A, Schoni-Affolter F, Burgisser P, Witteck A, Darling KE, Kovari H, Kaiser L, Evison JM, Elzi L, Gurter-De La Fuente V, Jost J, Moradpour D, Abravanel F, Izpopet J, Cavassini M; Data Center of the Swiss HIV Cohort Study, Lausanne, Switzerland (2011) Hepatitis E Virus seroprevalence and chronic infections in patients with HIV, Switzerland. Emerg Infect Dis 17:1074-1078

35. Moal V, Textoris J, Ben Amara A, Mehraj V, Berland Y, Colson P, Mege JL (2013) Chronic hepatitis E virus infection is specifically associated with an interferon-related transcriptional program. J Infect Dis 207:125-132

36. Suneetha PV, Pischke S, Schlaphoff V, Grabowski J, Fytili P, Gronert A, Bremer B, Markova A, Jaroszewicz J, Bara C, Manns MP, Cornberg M, Wedemeyer H (2012) Hepatitis E virus (HEV)-specific T-cell responses are associated with control of HEV infection. Hepatology 55:695-708

37. Barnaud E, Rogee S, Garry P, Rose N, Pavio N (2012) Thermal inactivation of infectious hepatitis $E$ virus in experimentally contaminated food. Appl Environ Microbiol 78:5153-5159

38. Charpin C, Mahe S, Keranflec'h A, Belloc C, Cariolet R, Le Potier MF, Rose N (2012) Infectiousness of pigs infected by the Porcine Reproductive and Respiratory Syndrome virus (PRRSV) is time-dependent. Vet Res 43:69

39. Satou K, Nishiura H (2007) Transmission dynamics of hepatitis E among swine: potential impact upon human infection. BMC Vet Res 3:9

40. Ihaka R, Gentleman R (1996) R: a language for data analysis and graphics. J Comp Graph Stat 5:299-314

41. Inc (2011) SI: SAS/STAT User's Guide. SAS Institute, Cary, NC USA

42. Gubbins S, Turner J, Baylis M, van der Stede Y, van Schaik G, Abrahantes JC, Wilson AJ (2014) Inferences about the transmission of Schmallenberg virus within and between farms. Prev Vet Med 116:380-390

43. Mateu E, Diaz I (2008) The challenge of PRRS immunology. Vet J 177:345-351

44. Diaz I, Darwich L, Pappaterra G, Pujols J, Mateu E (2005) Immune responses of pigs after experimental infection with a European strain of Porcine reproductive and respiratory syndrome virus. J Gen Virol 86:1943-1951

\section{Submit your next manuscript to BioMed Central and take full advantage of:}

- Convenient online submission

- Thorough peer review

- No space constraints or color figure charges

- Immediate publication on acceptance

- Inclusion in PubMed, CAS, Scopus and Google Scholar

- Research which is freely available for redistribution 